\title{
Semigroup-valued solutions of some composite equations
}

\author{
JACEK CHUDZIAK
}

Abstract. Let $X$ be a linear space over the field $K$ of real or complex numbers and $(S, \circ)$ be a semigroup. We determine all solutions of the functional equation

$$
f(x+g(x) y)=f(x) \circ f(y) \quad \text { for } \quad x, y \in X
$$

in the class of pairs of functions (f,g) such that $f: X \rightarrow S$ and $g: X \rightarrow K$ satisfies some regularity assumptions. Several consequences of this result are presented.

Mathematics Subject Classification (2000). 39B12, 39B52.

Keywords. Gołąb-Schinzel equation, semigroup-valued solution, algebraically interior point.

\section{Introduction}

Let $X$ be a linear space over the field $K$ of real or complex numbers. The solutions $f: X \rightarrow K$ of the Gołąb-Schinzel functional equation

$$
f(x+f(x) y)=f(x) f(y) \quad \text { for } \quad x, y \in X,
$$

have been intensively studied in the last half-century. Equation (1) is one of the most important equations of a composite type and plays a prominent role e.g. in the determination of substructures of various algebraical structures $[1$, pp. 311-319], [3,4]. The solutions of (1) and its further generalizations, namely

$$
f\left(x+f(x)^{n} y\right)=t f(x) f(y) \quad \text { for } \quad x, y \in X,
$$

where $n$ is a nonnegative integer and $t$ is a nonzero real number; and

$$
f(x+M(f(x)) y)=f(x) f(y) \quad \text { for } \quad x, y \in X,
$$

where $M: K \rightarrow K$, have been considered under various regularity assumptions e.g. in [2-4] and [19-21]. In the real case the functional equation

$$
f(x+M(f(x)) y)=f(x) \circ f(y) \quad \text { for } \quad x, y \in X,
$$


where $\circ$ is a binary operation on $\mathbb{R}$ satisfying some additional conditions (commutativity, associativity etc.), was studied in [5,7,16,25] and [26]. For more information concerning (1)-(4) and their further applications (e.g. to mathematical meteorology and fluid dynamics) we refer to the survey paper [6]. Various aspects of stability problems for the Gołąb-Schinzel functional equations were considered in $[8-13,15,17]$ and $[18]$. In the case where $(S, \circ)$ is an arbitrary semigroup, the general solution of the equation

$$
f(x+g(x) y)=f(x) \circ f(y) \quad \text { for } \quad x, y \in \mathbb{R}
$$

in the class of pairs $(f, g)$ such that $f: \mathbb{R} \rightarrow S$ and $g: \mathbb{R} \rightarrow \mathbb{R}$ is continuous, was determined in [14]. The functional equation

$$
f(x+g(x) y)=f(x) f(y) \quad \text { for } \quad x, y \in X
$$

was considered in [22] under the assumption that $f$ and $g$, mapping a real linear space $X$ into $\mathbb{R}$, are continuous on rays.

In the present paper we generalize substantially the results from [14] and [22] in various directions. Namely, we determine the general solution of the equation

$$
f(x+g(x) y)=f(x) \circ f(y) \quad \text { for } \quad x, y \in X
$$

in the case where $X$ is a linear space over the field $K$ of real or complex numbers, $(S, \circ)$ is an arbitrary semigroup, $f: X \rightarrow S$ and $g: X \rightarrow K$ satisfies some regularity assumptions. Several consequences of this result are presented, as well. In particular, applying our main result and using a natural correspondence between (5) and the pexiderized version of the Gołąb-Schinzel equation, that is

$$
F(x+G(x) y))=H(x) \circ K(y) \quad \text { for } \quad x, y \in X,
$$

we obtain a generalization of the results in [23].

In what follows $B(x, r)$ denotes the open ball (in $K$ ) with a center at $x \in K$ and a radius $r>0$. Let us recall $[24$, p. 596] that given a nonempty subset $A$ of $X$, we say that $a \in A$ is an algebraically interior point of $A$, provided, for every $x \in X \backslash\{0\}$, there is $r_{x}>0$ such that $a+B\left(0, r_{x}\right) x=\{a+b x: b \in$ $\left.B\left(0, r_{x}\right)\right\} \subset A$. By $\operatorname{int}_{a} A$ we denote a set of all algebraically interior points of $A$. If $f: X \rightarrow \mathbb{R}$ and $x \in X$ then a function $f_{x}: \mathbb{R} \rightarrow \mathbb{R}$ is given by $f_{x}(t)=f(t x)$ for $t \in \mathbb{R}$. Furthermore, given a nonempty subset $S_{0}$ of $S$, we put $Z_{L}\left(S_{0}\right):=\left\{s \in S: s \circ a=s\right.$ for $\left.a \in S_{0}\right\}$ and $Z\left(S_{0}\right):=\{s \in S: s \circ a=$ $a \circ s=s$ for $\left.a \in S_{0}\right\}$.

\section{Preliminary results}

Remark 1. Let $X$ be a linear space over the field $K$ of real or complex numbers and $(S, \circ)$ be a semigroup. Equation (5) has a solution if and only if $E(S):=$ 
$\{s \in S: s \circ s=s\} \neq \emptyset$. In fact, if $(f, g)$ is a solution of $(5)$, then $f(0)=$ $f(0) \circ f(0)$, so $f(0) \in E(S)$. Conversely, if $s \in E(S)$, then the pair $(f, g)$, where $g$ is an arbitrary function and $f \equiv s \in E(S)$, is a solution of (5).

Next, we present a result describing degenerate solutions of (5), i.e. such solutions $(f, g)$ that either $f$ or $g$ is constant.

Proposition 1. A pair of functions $(f, g)$ is a degenerate solution of (5) if and only if one of the following conditions is valid:

(i) there is an $s \in E(S)$ such that $f \equiv s$;

(ii) $g \equiv 0$ and there exists a subsemigroup $S_{0}$ of $S$ such that $u \circ v=u$ for $u, v \in S_{0}$ and $f(X) \subset S_{0}$;

(iii) $g \equiv 1$ and $f$ is a homomorphism of the additive group of $K$ into $(S, \circ)$.

Proof. It is clear that if one of the conditions $(i)-(i i i)$ holds, then $(f, g)$ is a degenerate solution of (5). So, assume that $(f, g)$ is a degenerate solution of (5). If $f$ is constant, then according to Remark 1 , we get $(i)$ with $s:=f(0)$. Now, assume that $f$ is nonconstant and $g$ is constant, say $g \equiv c$. If $c=0$, then (ii) holds with $S_{0}:=f(X)$. The case where $c=1$ leads to (iii). Suppose that $c \notin\{0,1\}$. Then, in view of (5), we obtain $f(x+c y)=f(x) \circ f(y)$ for $x, y \in X$, whence $f(c y)=f(0) \circ f(y)$ for $y \in X$. Therefore, for every $x, y \in X$, we get

$$
\begin{aligned}
f(y) & =f\left(c \frac{c x-y}{c^{2}-c}+c^{2} \frac{y-x}{c^{2}-c}\right)=f(0) \circ f\left(\frac{c x-y}{c^{2}-c}+c \frac{y-x}{c^{2}-c}\right) \\
& =f(0) \circ f\left(\frac{c x-y}{c^{2}-c}\right) \circ f\left(\frac{y-x}{c^{2}-c}\right)=f\left(c \frac{c x-y}{c^{2}-c}\right) \circ f\left(\frac{y-x}{c^{2}-c}\right) \\
& =f\left(c \frac{c x-y}{c^{2}-c}+c \frac{y-x}{c^{2}-c}\right)=f(x) .
\end{aligned}
$$

This yields a contradiction, because $f$ is nonconstant.

From now on we will deal only with the non-degenerate solutions of (5), i.e. with such solutions $(f, g)$ that neither $f$ nor $g$ is constant.

The following result plays a crucial role in our considerations.

Proposition 2. Let $X$ be a linear space over the field $K$ of real or complex numbers, $(S, \circ)$ be a semigroup, $f: X \rightarrow S$ and $g: X \rightarrow K$. Assume that $(f, g)$ is a non-degenerate solution of (5). Then each of the following regularity conditions:

$\left(C_{1}\right) \quad 0 \in g(X)$ and $\operatorname{int}_{a}\{x \in X \mid g(x) \neq 0\} \neq \emptyset$;

$\left(C_{2}\right) \operatorname{int}_{a}\{x \in X \mid g(x) \notin\{0,1\}\} \neq \emptyset$;

$\left(C_{3}\right) g$ is continuous on rays

implies that either there exists a nontrivial $K$-linear functional $L: X \rightarrow K$ such that 


$$
g(x)=L(x)+1 \quad \text { for } \quad x \in X,
$$

or there exists a nontrivial $\mathbb{R}$-linear functional $L: X \rightarrow \mathbb{R}$ such that

$$
g(x)=\max \{L(x)+1,0\} \quad \text { for } \quad x \in X .
$$

Proof. In view of (5), for every $x, y, z \in X$, we have

$$
(f(x) \circ f(y)) \circ f(z)=f(x+g(x) y) \circ f(z)=f(x+g(x) y+g(x+g(x) y) z)
$$

and

$$
f(x) \circ(f(y) \circ f(z))=f(x) \circ f(y+g(y) z)=f(x+g(x) y+g(x) g(y) z) .
$$

Thus, as $\circ$ is associative, we get

$$
f(x+g(x) y+g(x+g(x) y) z)=f(x+g(x) y+g(x) g(y) z) \text { for } x, y, z \in X .
$$

Therefore, if $g(x+g(x) y)=0$ for some $x, y \in X$ then

$$
f(x+g(x) y)=f(x+g(x) y+g(x) g(y) z) \text { for } z \in X
$$

and so, as $f$ is nonconstant, we get $g(x) g(y)=0$. Similarly if, for some $x, y \in X$, $g(x) g(y)=0$ then

$$
f(x+g(x) y+g(x+g(x) y) z)=f(x+g(x) y) \text { for } z \in X .
$$

Since $f$ is nonconstant, this means that $g(x+g(x) y)=0$. In this way we have proved that, for every $x, y \in X$, it holds that

$$
g(x+g(x) y)=0 \quad \text { if and only if } g(x) g(y)=0 .
$$

So, if $\left(C_{1}\right)$ is valid, according to [17, Theorem 1$]$, we obtain that

$$
g(x+g(x) y)=g(x) g(y) \quad \text { for } \quad x, y \in X .
$$

Hence, applying [4, Theorem 3], we get the assertion.

Now, assume that $\left(C_{2}\right)$ holds. We show that $0 \in g(X)$. Suppose that $0 \notin g(X)$. Then, by (5), we get

$$
f(0)=f\left(x+g(x)\left(-\frac{x}{g(x)}\right)\right)=f(x) \circ f\left(-\frac{x}{g(x)}\right) \quad \text { for } \quad x \in X .
$$

Therefore, taking $x \in G_{1}:=\{x \in X \mid g(x) \neq 1\}$ and $y=\frac{x}{1-g(x)}$, we have $x+g(x) y=y$, so by (5) and (11), we obtain

$$
\begin{aligned}
f(x) & =f(x) \circ f(0)=f(x) \circ f(y) \circ f\left(-\frac{y}{g(y)}\right) \\
& =f(x+g(x) y) \circ f\left(-\frac{y}{g(y)}\right)=f(y) \circ f\left(-\frac{y}{g(y)}\right)=f(0) .
\end{aligned}
$$

Thus

$$
f(x)=f(0) \quad \text { for } \quad x \in G_{1} .
$$


Next, as $(f, g)$ is a non-degenerate solution of (5), there is $x \in X \backslash\{0\}$ such that $f_{x}$ is a nonconstant function. Fix $a \in \operatorname{int}_{a}\{x \in X \mid g(x) \notin\{0,1\}\}=\operatorname{int}_{a} G_{1}$. Then, in view of (12), $f(a)=f(0)$. Furthermore, let $r_{x}>0$ be such that

$$
a+B\left(0, r_{x}\right) x \subset G_{1} .
$$

We claim that there is $r>0$ such that

$$
f(B(0, r) x)=\{f(0)\} .
$$

Suppose that (14) does not hold. Then there exists a sequence $\left(t_{n}\right)$ of elements of $K$ converging to 0 and such that $f\left(t_{n} x\right) \neq f(0)$ for $n \in \mathbb{N}$. Then, by (12), $g\left(t_{n} x\right)=1$ for $n \in \mathbb{N}$ and so, in view of (5), for every $n \in \mathbb{N}$, we get $f\left(a+t_{n} x\right)=f\left(t_{n} x+g\left(t_{n} x\right) a\right)=f\left(t_{n} x\right) \circ f(a)=f\left(t_{n} x\right) \circ f(0)=f\left(t_{n} x\right) \neq f(0)$.

On the other hand, from (13) it follows that $a+t_{n} x \in G_{1}$ for sufficiently large $n \in \mathbb{N}$. Hence, by (12), $f\left(a+t_{n} x\right)=f(0)$ for sufficiently large $n \in \mathbb{N}$, which yields a contradiction. In this way we have proved (14). Since the function $f_{x}$ is nonconstant, there is $k \in K$ with $f(k x)=f_{x}(k) \neq f_{x}(0)=f(0)$. Therefore, making use of (12), we get

$$
g(k x)=1 .
$$

Now, we show by induction that for every $b \in B(0, r)$ and $n \in \mathbb{N}$ it holds that

$$
f((k+n b) x) \neq f(0) .
$$

Note that by (5), (14) and (15), for every $b \in B(0, r)$, we have $f((k+b) x)=f(k x+g(k x) b x)=f(k x) \circ f(b x)=f(k x) \circ f(0)=f(k x) \neq f(0)$.

Thus, (16) is valid for $n=1$. Next, fix $n \in \mathbb{N}$ and assume that (16) holds for every $b \in B(0, r)$. Then, in view of (12), $g((k+b n) x)=1$, so applying (5), (14) and (16), for every $b \in B(0, r)$, we obtain

$$
\begin{aligned}
f((k+(n+1) b) x) & =f((k+n b) x+b x)=f((k+n b) x+g((k+n b) x) b x) \\
& =f((k+n b) x) \circ f(b x)=f((k+n b) x) \circ f(0) \\
& =f((k+n b) x) \neq f(0) .
\end{aligned}
$$

In this way we have proved that (16) holds for every $b \in B(0, r)$ and $n \in \mathbb{N}$. Note however that, taking $n_{0} \in \mathbb{N}$ with $\frac{k}{n_{0}} \in B(0, r)$, we have

$$
f\left(k x+n_{0}\left(-\frac{k}{n_{0}}\right) x\right)=f(0) .
$$

This yields a contradiction and shows that $0 \in g(X)$. Since by $\left(C_{2}\right)$

$$
\emptyset \neq \operatorname{int}_{a}\{x \in X \mid g(x) \notin\{0,1\}\} \subset \operatorname{int}_{a}\{x \in X \mid g(x) \neq 0\},
$$

applying [17, Theorem 1], from (9) we deduce (10). Thus, according to [4, Theorem 3], we get the assertion. 
Finally, if $\left(C_{3}\right)$ holds for $n$, as in the previous case, we get that the function $f_{x}$ is nonconstant for some $x \in X \backslash\{0\}$. Moreover, by $\left(C_{3}\right), g_{x}$ is continuous and, in view of (5),

$f_{x}\left(t+g_{x}(t) s\right)=f(t x+g(t x) s x)=f(t x) \circ f(s x)=f_{x}(t) \circ f_{x}(s)$ for $s, t \in \mathbb{R}$.

So, applying $[14$, Lemma 1$]$, we obtain that $g_{x}\left(t_{0}\right)=0$ for some $t_{0} \in \mathbb{R}$, that is $0 \in g_{x}(\mathbb{R}) \subset g(X)$. Note also that by [14, Lemma 2], we have $g(0)=$ $g_{x}(0)=1$. Thus, in view of $\left(C_{3}\right), 0 \in \operatorname{int}_{a}\{x \in X \mid g(x) \neq 0\}$. Consequently, applying [17, Theorem 1], from (9) we derive (10). Therefore, according to $[4$, Theorem 3], we get the assertion.

\section{Main results}

The next theorem is the main result of the paper.

Theorem 1. Assume that $X$ is a linear space over the field $K$ of real or complex numbers, $(S, \circ)$ is a semigroup, $f: X \rightarrow S, g: X \rightarrow K$ and one of the conditions $\left(C_{1}\right)-\left(C_{3}\right)$ holds. A pair of functions $(f, g)$ is a non-degenerate solution of (5) if and only if one of the following two cases holds:

(i) there exist a nontrivial $K$-linear functional $L: X \rightarrow K$ and functions $a: X \rightarrow S$ and $\phi: K \rightarrow S$ satisfying the conditions

$$
\begin{gathered}
a(x+y)=a(x) \circ a(y) \quad \text { for } \quad x, y \in X, \\
\phi(s t)=\phi(s) \circ \phi(t) \quad \text { for } \quad s, t \in K,
\end{gathered}
$$

and

$$
\phi(t) \circ a(x)=a(t x) \circ \phi(t) \quad \text { for } \quad x \in X, t \in K
$$

such that $g$ is of the form (7) and

$$
f(x)=a(x) \circ \phi(L(x)+1) \quad \text { for } \quad x \in X
$$

(ii) there exist a nontrivial $\mathbb{R}$-linear functional $L: X \rightarrow \mathbb{R}$, functions $a: X \rightarrow S$ and $\phi:[0, \infty) \rightarrow S$ satisfying (17),

$$
\begin{aligned}
\phi(s t) & =\phi(s) \circ \phi(t) \quad \text { for } \quad s, t \in[0, \infty), \\
a(t x) \circ \phi(t) & =\phi(t) \circ a(x) \quad \text { for } \quad x \in X, t \in[0, \infty)
\end{aligned}
$$

and $l \in Z_{L}(\phi([0, \infty))) \cap E(S)$ such that $g$ is of the form (8) and

$$
f(x)= \begin{cases}a(x) \circ \phi(L(x)+1) & \text { whenever } L(x)+1 \geq 0 \\ a(x) \circ \phi(-(L(x)+1)) \circ l & \text { otherwise. }\end{cases}
$$


Proof. Assume that a pair $(f, g)$ is a non-degenerate solution of (5). Then, according to Proposition 2, $g$ is either of the form (7) or (8). In the first case, let us fix $x_{0} \in X \backslash \operatorname{ker} L$ and define the functions $\Pi_{1}, \Pi_{2}: X \rightarrow X$ by

$$
\Pi_{1}(x)=x-\frac{L(x)}{L\left(x_{0}\right)} x_{0} \quad \text { for } \quad x \in X
$$

and

$$
\Pi_{2}(x)=\frac{L(x)}{L\left(x_{0}\right)} x_{0} \quad \text { for } \quad x \in X .
$$

Then $\Pi_{1}$ and $\Pi_{2}$ are linear and $\Pi_{1}(x)+\Pi_{2}(x)=x$ for $x \in X$. Moreover $L\left(\Pi_{1}(x)\right)=0$ for $x \in X$, which yields that

$$
g\left(\Pi_{1}(x)\right)=1 \quad \text { for } \quad x \in X .
$$

Therefore, taking $a: X \rightarrow S$ of the form

$$
a(x)=f\left(\Pi_{1}(x)\right) \text { for } \quad x \in X
$$

and $\phi: K \rightarrow S$ of the form

$$
\phi(t)=f\left(\frac{t-1}{L\left(x_{0}\right)} x_{0}\right) \quad \text { for } \quad t \in K,
$$

in view of (5), we obtain

$$
\begin{aligned}
a(x+y) & =f\left(\Pi_{1}(x+y)\right)=f\left(\Pi_{1}(x)+\Pi_{1}(y)\right)=f\left(\Pi_{1}(x)+g\left(\Pi_{1}(x)\right) \Pi_{1}(y)\right) \\
& =f\left(\Pi_{1}(x)\right) \circ f\left(\Pi_{1}(y)\right)=a(x) \circ a(y) \quad \text { for } \quad x, y \in X
\end{aligned}
$$

and, since $s t-1=s-1+((s-1)+1)(t-1)$,

$$
\begin{aligned}
\phi(s t) & =f\left(\frac{s t-1}{L\left(x_{0}\right)} x_{0}\right)=f\left(\frac{s-1}{L\left(x_{0}\right)} x_{0}+\left(L\left(\frac{s-1}{L\left(x_{0}\right)} x_{0}\right)+1\right) \frac{t-1}{L\left(x_{0}\right)} x_{0}\right) \\
& =f\left(\frac{s-1}{L\left(x_{0}\right)} x_{0}+g\left(\frac{s-1}{L\left(x_{0}\right)} x_{0}\right) \frac{t-1}{L\left(x_{0}\right)} x_{0}\right) \\
& =f\left(\frac{s-1}{L\left(x_{0}\right)} x_{0}\right) \circ f\left(\frac{t-1}{L\left(x_{0}\right)} x_{0}\right) \\
& =\phi(s) \circ \phi(t) \quad \text { for } s, t \in K .
\end{aligned}
$$

Moreover, for every $x \in X$, we get

$f(x)=f\left(\Pi_{1}(x)+\Pi_{2}(x)\right)=f\left(\Pi_{1}(x)+g\left(\Pi_{1}(x)\right) \Pi_{2}(x)\right)=f\left(\Pi_{1}(x)\right) \circ f\left(\Pi_{2}(x)\right)$.

Hence by (25) and (27), we obtain

$$
f(x)=a(x) \circ f\left(\frac{L(x)}{L\left(x_{0}\right)} x_{0}\right) \quad \text { for } \quad x \in X .
$$

Thus, taking (28) into account, we conclude that (20) holds. It remains to show (19). To this end note that, in view of (26), for every $x \in X$ and $t \in K$, we have 


$$
\begin{aligned}
\Pi_{1}(t x)+g\left(\Pi_{1}(t x)\right) \frac{t-1}{L\left(x_{0}\right)} x_{0} & =\frac{t-1}{L\left(x_{0}\right)} x_{0}+\left(\frac{t-1}{L\left(x_{0}\right)} L\left(x_{0}\right)+1\right) \Pi_{1}(x) \\
& =\frac{t-1}{L\left(x_{0}\right)} x_{0}+\left(L\left(\frac{t-1}{L\left(x_{0}\right)} x_{0}\right)+1\right) \Pi_{1}(x) \\
& =\frac{t-1}{L\left(x_{0}\right)} x_{0}+g\left(\frac{t-1}{L\left(x_{0}\right)} x_{0}\right) \Pi_{1}(x) .
\end{aligned}
$$

Hence, by (5), we obtain

$$
f\left(\Pi_{1}(t x)\right) \circ f\left(\frac{t-1}{L\left(x_{0}\right)} x_{0}\right)=f\left(\frac{t-1}{L\left(x_{0}\right)} x_{0}\right) \circ f\left(\Pi_{1}(x)\right) \quad \text { for } \quad x \in X, t \in K .
$$

So, taking (27) and (28) into account, we get (19). In this way we have proved that $(i)$ is valid.

Next consider the case where $g$ is of the form (8). Let $x_{0} \in X \backslash \operatorname{ker} L$ and let $\Pi_{1}, \Pi_{2}: X \rightarrow X$ be given by (24) and (25), respectively. Furthermore, let $a: X \rightarrow S$ be of the form $(27)$ and $\phi:[0, \infty) \rightarrow S$ be given by

$$
\phi(t)=f\left(\frac{t-1}{L\left(x_{0}\right)} x_{0}\right) \quad \text { for } \quad t \in[0, \infty) .
$$

Then, arguing as in the previous case, we obtain (17), (21), (22) and (29). In particular, by (29) and (30), we have

$$
f(x)=a(x) \circ \phi(L(x)+1) \quad \text { whenever } \quad L(x)+1 \geq 0 .
$$

Fix $x \in X$ with $L(x)+1<0$. Then

$$
L\left(\frac{-(L(x)+2)}{L\left(x_{0}\right)} x_{0}\right)+1=-(L(x)+1)>0,
$$

so by $(8)$, we get $g\left(\frac{-(L(x)+2)}{L\left(x_{0}\right)} x_{0}\right)=-(L(x)+1)$. Therefore, making use of (5), (31) and (32), we obtain

$$
\begin{aligned}
f\left(\frac{L(x)}{L\left(x_{0}\right)} x_{0}\right) & =f\left(\frac{-(L(x)+2)}{L\left(x_{0}\right)} x_{0}+g\left(\frac{-(L(x)+2)}{L\left(x_{0}\right)} x_{0}\right)\left(\frac{-2 x_{0}}{L\left(x_{0}\right)}\right)\right) \\
& =f\left(\frac{-(L(x)+2)}{L\left(x_{0}\right)} x_{0}\right) \circ f\left(\frac{-2 x_{0}}{L\left(x_{0}\right)}\right) \\
& =a\left(\frac{-(L(x)+2)}{L\left(x_{0}\right)} x_{0}\right) \circ \phi(-(L(x)+1)) \circ f\left(\frac{-2 x_{0}}{L\left(x_{0}\right)}\right) .
\end{aligned}
$$

On the other hand, in view of $(24)$, we get $\Pi_{1}(0)=0$ and $\Pi_{1}\left(\frac{-(L(x)+2)}{L\left(x_{0}\right)} x_{0}\right)$ $=0$, whence by $(27), a\left(\frac{-(L(x)+2)}{L\left(x_{0}\right)} x_{0}\right)=f(0)=a(0)$. Thus, for every $x \in X$ with $L(x)+1<0$, we have

$$
f\left(\frac{L(x)}{L\left(x_{0}\right)} x_{0}\right)=a(0) \circ \phi(-(L(x)+1)) \circ f\left(\frac{-2 x_{0}}{L\left(x_{0}\right)}\right)
$$


and so, by (17),

$$
a(x) \circ f\left(\frac{L(x)}{L\left(x_{0}\right)} x_{0}\right)=a(x) \circ \phi(-(L(x)+1)) \circ f\left(\frac{-2 x_{0}}{L\left(x_{0}\right)}\right) .
$$

Hence, taking (29) into account, we conclude that

$$
f(x)=a(x) \circ \phi(-(L(x)+1)) \circ l \quad \text { whenever } L(x)+1<0,
$$

where $l:=f\left(\frac{-2 x_{0}}{L\left(x_{0}\right)}\right)$. Note also that $L\left(\frac{-2 x_{0}}{L\left(x_{0}\right)}\right)+1=-1<0$, so $g\left(\frac{-2 x_{0}}{L\left(x_{0}\right)}\right)=0$. Thus, in view of (5), for every $x \in X$, it holds that

$l \circ f(x)=f\left(\frac{-2 x_{0}}{L\left(x_{0}\right)}\right) \circ f(x)=f\left(\frac{-2 x_{0}}{L\left(x_{0}\right)}+g\left(\frac{-2 x_{0}}{L\left(x_{0}\right)}\right) x\right)=f\left(\frac{-2 x_{0}}{L\left(x_{0}\right)}\right)=l$.

Hence $l \in Z_{L}(f(X))$. Since $l \in f(X)$ and, by $(30), \phi([0, \infty)) \subset f(X)$, this implies that $l \in Z_{L}(\phi([0, \infty))) \cap E(S)$. Therefore, taking (31) and (33) into account, we get (23). Consequently (ii) holds.

Since the converse is easy to check, the proof is completed.

In the case of a commutative semigroup, a description of the solutions of (5) is significantly simpler. Namely, we have the following result.

Proposition 3. Let $X$ be a linear space over the field $K$ of real or complex numbers, $(S, \circ)$ be a commutative semigroup, $f: X \rightarrow S, g: X \rightarrow K$ and assume that one of the conditions $\left(C_{1}\right)-\left(C_{3}\right)$ holds. Then a pair $(f, g)$ is a non-degenerate solution of (5) if and only if one of the subsequent cases holds:

(a) there exist a nontrivial $K$-linear functional $L: X \rightarrow K$ and a nontrivial homomorphism $\psi$ of the multiplicative semigroup of $K$ into $(S, \circ)$ such that $g$ is of the form (7) and

$$
f(x)=\psi(L(x)+1) \quad \text { for } \quad x \in X ;
$$

(b) there exist a nontrivial $\mathbb{R}$-linear functional $L: X \rightarrow \mathbb{R}$, a homomorphism $\psi$ of the multiplicative semigroup of nonnegative real numbers into $(S, \circ)$ and $z \in Z(\psi([0, \infty)))$ such that $\psi \neq z, g$ is of the form (8) and

$$
f(x)= \begin{cases}\psi(L(x)+1) & \text { whenever } L(x)+1 \geq 0 \\ z & \text { otherwise. }\end{cases}
$$

Proof. Assume that a pair $(f, g)$ is a non-degenerate solution of (5). Then one of the conditions $(i),(i i)$ of Theorem 1 holds. In the case of $(i),(19)$ and the commutativity of $\circ$ imply that $a(2 x) \circ \phi(2)=a(x) \circ \phi(2)$ for $x \in X$, whence by (18), $a(2 x) \circ \phi(1)=a(x) \circ \phi(1)$ for $x \in X$. Thus, in view of (17), for every $x \in X$, we get

$$
a(x) \circ \phi(1)=a(-x) \circ a(2 x) \circ \phi(1)=a(-x) \circ a(x) \circ \phi(1)=a(0) \circ \phi(1) .
$$


Therefore, taking $\psi:=a(0) \circ \phi$, by (17), (18), (20) and (36), for every $x \in X$, we obtain

$$
\begin{aligned}
f(x) & =a(x) \circ \phi(L(x)+1)=a(x) \circ a(0) \circ \phi(1) \circ \phi(L(x)+1) \\
& =a(x) \circ \phi(1) \circ a(0) \circ \phi(L(x)+1)=a(0) \circ \phi(1) \circ a(0) \circ \phi(L(x)+1) \\
& =a(0) \circ \phi(L(x)+1) . \\
& =\psi(L(x)+1) .
\end{aligned}
$$

Moreover, by (17), $a(0)=a(0) \circ a(0)$, so using (18) one can easily check that $\psi$ is a homomorphism of the multiplicative semigroup of $K$ into $(S, \circ)$. Note also that, as $f$ is nonconstant, $\psi$ is nontrivial. In this way we have proved that $(a)$ holds.

Next assume that condition (ii) of Theorem 1 holds. Then, as in the previous case, we obtain that (36) is valid, $\psi:=a(0) \circ \phi$ is a homomorphism of the multiplicative semigroup of $[0, \infty)$ into $(S, \circ)$ and $f(x)=\psi(L(x)+1)$ whenever $L(x)+1 \geq 0$. Furthermore, the commutativity of $\circ$ implies that $z:=a(0) \circ l \in Z(\psi([0, \infty)))$. Hence, as $(S, \circ)$ is commutative, by (17), (18), (23) and (36), for every $x \in X$ with $L(x)+1<0$, we get

$$
\begin{gathered}
f(x)=a(x) \circ \phi(-(L(x)+1)) \circ l=a(x) \circ a(0) \circ a(0) \circ \phi(1) \circ \phi(-(L(x)+1)) \circ l \\
=a(x) \circ \phi(1) \circ a(0) \circ l \circ a(0) \circ \phi(-(L(x)+1)) \\
=a(0) \circ \phi(1) \circ z \circ \psi(-(L(x)+1))=\psi(1) \circ z=z .
\end{gathered}
$$

Thus $f$ is of the form (35) and therefore $(b)$ is valid.

The converse is easy to check, so the proof is completed.

The next result concerns the case where $X=\mathbb{R}$.

Proposition 4. Assume that $(S, \circ)$ is a semigroup, $f: \mathbb{R} \rightarrow S, g: \mathbb{R} \rightarrow \mathbb{R}$ are nonconstant functions and int $\{x \in \mathbb{R} \mid g(x) \notin\{0,1\}\} \neq \emptyset$. Then a pair of functions $(f, g)$ satisfies (5) if and only if one of the following two conditions holds:

(a) there exist a nontrivial homomorphism $\psi: \mathbb{R} \rightarrow S$ of the multiplicative semigroup of real numbers into $(S, \circ)$ and $c \in \mathbb{R} \backslash\{0\}$ such that

$$
\begin{aligned}
& g(x)=c x+1 \quad \text { for } \quad x \in \mathbb{R}, \\
& f(x)=\psi(c x+1) \quad \text { for } \quad x \in \mathbb{R} ;
\end{aligned}
$$

(b) there exist a nontrivial homomorphism $\psi:[0, \infty) \rightarrow S$ of the multiplicative semigroup of nonnegative real numbers into $(S, \circ), c \in \mathbb{R} \backslash\{0\}$ and $z \in Z(\psi([0, \infty))) \cap E(S)$ such that

$$
\begin{aligned}
& g(x)=\max \{c x+1,0\} \quad \text { for } \quad x \in \mathbb{R}, \\
& f(x)= \begin{cases}\psi(c x+1) & \text { whenever } c x+1 \geq 0 \\
z & \text { otherwise. }\end{cases}
\end{aligned}
$$


Proof. Assume that a pair $(f, g)$ satisfies (5). Since $f$ and $g$ are nonconstant and $\operatorname{int}\{x \in X \mid g(x) \notin\{0,1\}\} \neq \emptyset$, the assumptions of Theorem 1 are satisfied with $X=\mathbb{R}$. So, one of the conditions $(i)$ or $(i i)$ of Theorem 1 holds. In the case of $(i)$, we have $L(x)=c x$ for $x \in \mathbb{R}$ with some $c \in \mathbb{R} \backslash\{0\}$. Let

$$
\psi(t):=a\left(-\frac{1}{c}\right) \circ \phi(t) \circ a\left(\frac{1}{c}\right) \quad \text { for } \quad t \in \mathbb{R} .
$$

Since, by (19), $a(0) \circ \phi(t)=\phi(t) \circ a(0)$ for $t \in X$ and $\phi: \mathbb{R} \rightarrow S$ is a homomorphism of the multiplicative semigroup of real numbers into $(S, \circ)$, making use of (17), we obtain

$$
\begin{aligned}
\psi(s t) & =a\left(-\frac{1}{c}\right) \circ \phi(s t) \circ a\left(\frac{1}{c}\right)=a\left(-\frac{1}{c}\right) \circ \phi(s) \circ \phi(t) \circ a\left(\frac{1}{c}\right) \\
& =a\left(-\frac{1}{c}\right) \circ \phi(s) \circ \phi(t) \circ a(0) \circ a\left(\frac{1}{c}\right) \\
& =a\left(-\frac{1}{c}\right) \circ \phi(s) \circ a(0) \circ \phi(t) \circ a\left(\frac{1}{c}\right) \\
& =a\left(-\frac{1}{c}\right) \circ \phi(s) \circ a\left(\frac{1}{c}\right) \circ a\left(-\frac{1}{c}\right) \circ \phi(t) \circ a\left(\frac{1}{c}\right) \\
& =\psi(s) \circ \psi(t) \quad \text { for } \quad s, t \in \mathbb{R} .
\end{aligned}
$$

Hence $\psi$ is a homomorphism of the multiplicative semigroup of real numbers into $(S, \circ)$. Moreover, by (17) and (37), we get $\phi(t)=a\left(\frac{1}{c}\right) \circ \psi(t) \circ a\left(-\frac{1}{c}\right)$ for $t \in \mathbb{R}$. Thus, as $\phi$ is nontrivial, so is $\psi$. Furthermore, considering (17), (19) and (20), we have

$$
\begin{aligned}
f(x) & =a(x) \circ \phi(c x+1)=a\left(-\frac{1}{c}\right) \circ a\left(\frac{1}{c}(c x+1)\right) \circ \phi(c x+1) \\
& =a\left(-\frac{1}{c}\right) \circ \phi(c x+1) \circ a\left(\frac{1}{c}\right)=\psi(c x+1) \quad \text { for } \quad x \in \mathbb{R} .
\end{aligned}
$$

In this way we have proved that $(a)$ holds.

In the case of $(i i)$, similar arguments lead to $(b)$. As the converse is easy to check, this completes the proof.

Remark 2. Proposition 4 together with Proposition 1 generalizes [14, Theorem 1]. Note that in [14, Theorem 1] and [14, Corollary 1] in the formulae " $l \in Z_{L}(\phi([0, \infty))) \cap E(S)$ " and " $l \in Z(\psi([0, \infty))) \cap E(S)$ " the term " $\cap E(S)$ " is missing.

In general, the function $a$ acting in the assertion of Theorem 1 need not be constant. 
Example 1. Let $X$ be a real linear space of dimension at least 2 and let $S=\mathbb{R}^{2}$ be endowed with the following binary operation

$$
\left(x_{1}, y_{1}\right) \circ\left(x_{2}, y_{2}\right)=\left(x_{1} y_{1}, x_{1} y_{2}+y_{1}\right) \quad \text { for } \quad\left(x_{1}, y_{1}\right),\left(x_{2}, y_{2}\right) \in S \text {. }
$$

Define the functions $a: X \rightarrow S$ and $\phi: \mathbb{R} \rightarrow S$ by $a(x)=(1, x)$ for $x \in X$ and $\phi(t)=(t, 0)$ for $t \in \mathbb{R}$, respectively. Then an easy calculation shows that (17)-(19) hold. So, taking a nontrivial $\mathbb{R}$-linear functional $L: X \rightarrow \mathbb{R}$ and applying Theorem $1(i)$, we conclude that a pair of functions $(f, g)$, where $g: X \rightarrow \mathbb{R}$ is of the form (7) and $f: X \rightarrow S$ is given by

$$
f(x)=a(x) \circ \phi(L(x)+1)=(L(x)+1, x) \text { for } \quad x \in X,
$$

satisfies (5). Note also that $f$ is injective and, as $\operatorname{dim} X \geq 2, L$ is not. Thus, $f$ can not be represented in the form $f(x)=\psi(L(x)+1)$ for $x \in X$ with some $\psi: \mathbb{R} \rightarrow S$.

Next, we present the result concerning the solutions of (5) in the case where $(S, \circ)$ is a group.

Proposition 5. Let $X$ be a linear space over the field $K$ of real or complex numbers and let $(S, \circ)$ be a group (with unit element $e$ ). Assume that $f$ : $X \rightarrow S, g: X \rightarrow K$ and that one of the conditions $\left(C_{1}\right)-\left(C_{3}\right)$ holds. Then a pair $(f, g)$ satisfies (5) if and only if either $f=e$, or $g=1$ and $f$ is a homomorphism of an additive group $(X,+)$ into $(S, \circ)$.

Proof. Suppose that $(f, g)$ is a non-degenerate solution of (5). Then one of the conditions $(i)$ or $(i i)$ of Theorem 1 holds. In the first case, putting $t=0$ in (18) and considering the fact that $(S, \circ)$ is a group, we conclude that $\phi$ is constant. Furthermore, applying (19) with $t=0$, we get $\phi(0) \circ a(x)=$ $a(0) \circ \phi(0)$ for $x \in X$, so also $a$ is constant. Hence, in view of (20), $f$ is constant, which yields a contradiction. In the case where condition (ii) of Theorem 1 holds, the same arguments yield a contradiction (note that in this case $\phi=e, Z_{L}(\phi([0, \infty))) \cap E(S)=\{e\}$ and so $\left.l=e\right)$.

In this way we have proved that Eq. (5) has only degenerate solutions. Furthermore, as $(S, \circ)$ is a group, we have $E(S)=\{e\}$. Moreover, the only subsemigroup $S_{0}$ of $(S, \circ)$ such that $u \circ v=u$ for $u, v \in S_{0}$, is $S_{0}=\{e\}$. Therefore, applying Proposition 1, we obtain the assertion.

We complete the paper with two results concerning (6), which generalize some results in [23].

Proposition 6. Let $X$ be a linear space over the field $K$ of real or complex numbers and let $(S, \circ)$ be a commutative semigroup with unit element e. Assume that $F, H, K: X \rightarrow S, G: X \rightarrow K, F$ and $G$ are nonconstant and the set $F(X)$ contains at least one invertible element. Then a quadruple $(F, G, H, K)$ 
satisfies equation (6) if and only if there exist $x_{0} \in X, s, t \in S, k \in K \backslash\{0\}$ and functions $f: X \rightarrow S, g: X \rightarrow K$ such that a pair $(f, g)$ satisfies (5) and

$$
\begin{aligned}
& F(x)=s \circ t \circ f\left(x-x_{0}\right) \quad \text { for } \quad x \in X, \\
& G(x)=k g\left(x-x_{0}\right) \quad \text { for } \quad x \in X, \\
& H(x)=t \circ f\left(x-x_{0}\right) \quad \text { for } \quad x \in X, \\
& K(x)=s \circ f(k x) \quad \text { for } \quad x \in X .
\end{aligned}
$$

Proof. Assume that a quadruple $(F, G, H, K)$ satisfies Eq. (6). By the assumption, there is $x_{0} \in X$ such that $F\left(x_{0}\right) \circ p=p \circ F\left(x_{0}\right)=e$ for some $p \in S$. In (6) taking $y=0$ and next $x=x_{0}$, we get

$$
F(x)=H(x) \circ K(0) \quad \text { for } \quad x \in X
$$

and

$$
F\left(x_{0}+G\left(x_{0}\right) y\right)=H\left(x_{0}\right) \circ K(y) \quad \text { for } \quad y \in X,
$$

respectively. Since, in view of (42),

$$
F\left(x_{0}\right)=H\left(x_{0}\right) \circ K(0),
$$

using the commutativity of $\circ$, we derive that

$H(x)=H(x) \circ e=H(x) \circ F\left(x_{0}\right) \circ p=H\left(x_{0}\right) \circ p \circ H(x) \circ K(0)$ for $\quad x \in X$.

So considering (42), we get

$$
H(x)=H\left(x_{0}\right) \circ p \circ F(x) \text { for } \quad x \in X .
$$

In a similar way, using (43), we obtain

$$
K(x)=K(0) \circ p \circ F\left(x_{0}+G\left(x_{0}\right) x\right) \quad \text { for } \quad x \in X .
$$

Furthermore, by (6) and (44), for every $x, y \in X$, we get

$$
\begin{aligned}
F(x+G(x) y)) & =F(x+G(x) y)) \circ e=F(x+G(x) y)) \circ F\left(x_{0}\right) \circ p \\
& =p \circ H(x) \circ K(0) \circ H\left(x_{0}\right) \circ K(y) .
\end{aligned}
$$

Hence, in view of (42) and (43), we obtain

$$
F(x+G(x) y))=p \circ F(x) \circ F\left(x_{0}+G\left(x_{0}\right) y\right) \quad \text { for } \quad x, y \in X .
$$

Note also that $G\left(x_{0}\right) \neq 0$. Otherwise, as $G$ is nonconstant, In (45) putting $y=y_{1}$ with $G\left(y_{1}\right) \neq 0$, we would have that $F$ is constant, which yields a contradiction. Now, let

$$
f(x)=p \circ F\left(x+x_{0}\right) \quad \text { for } \quad x \in X
$$

and

$$
g(x)=\frac{G\left(x+x_{0}\right)}{G\left(x_{0}\right)} \quad \text { for } \quad x \in X .
$$


Then, making use of (47), we obtain

$$
\begin{aligned}
f(x+g(x) y) & =p \circ F\left(x_{0}+x+G\left(x+x_{0}\right) \frac{y}{G\left(x_{0}\right)}\right) \\
& =p \circ F\left(x_{0}+x\right) \circ p \circ F\left(x_{0}+G\left(x_{0}\right) \frac{y}{G\left(x_{0}\right)}\right) \\
& =f(x) \circ f(y) \text { for } x, y \in X,
\end{aligned}
$$

so a pair $(f, g)$ satisfies (5). Moreover, considering (44)-(46), (48) and (49), we obtain (38)-(41) with $k:=G\left(x_{0}\right), s:=K(0)$ and $t:=H\left(x_{0}\right)$.

Conversely, assume that (38)-(41) hold with some $k \in K \backslash\{0\}, x_{0} \in X$, $s, t \in S$ and functions $f: X \rightarrow S, g: X \rightarrow K$ such that the pair $(f, g)$ satisfies (5). Then, for every $x, y \in X$, we have

$$
\begin{aligned}
F(x+G(x) y)) & \left.=s \circ t \circ f\left(x-x_{0}+k g\left(x-x_{0}\right) y\right)\right) \\
& =s \circ t \circ f\left(x-x_{0}\right) \circ f(k y)=H(x) \circ K(y) .
\end{aligned}
$$

Proposition 7. Let $X$ be a linear space over the field $K$ of real or complex numbers, $(S, \circ)$ be a commutative semigroup with unit element $e$ and let $F, H, K$ : $X \rightarrow S, G: X \rightarrow K$. Assume that $F$ and $G$ are nonconstant, $G$ satisfies one of the conditions $\left(C_{1}\right)-\left(C_{3}\right)$ and the set $F(X)$ contains at least one invertible element. Then the quadruple $(F, G, H, K)$ satisfies Eq. (6) if and only if one of the following two cases holds:

(i) there exist a nontrivial $K$-linear functional $L: X \rightarrow K$ and a nontrivial homomorphism $\psi$ of the multiplicative semigroup of $K$ into $(S, \circ), s, t$ $\in S, k \in K \backslash\{0\}$ and $l \in K$ such that

$$
\begin{aligned}
& F(x)=s \circ t \circ \psi(L(x)+l) \quad \text { for } \quad x \in X, \\
& G(x)=k(L(x)+l) \quad \text { for } \quad x \in X, \\
& H(x)=t \circ \psi(L(x)+l) \quad \text { for } \quad x \in X, \\
& K(x)=s \circ \psi(L(k x)+1) \quad \text { for } \quad x \in X
\end{aligned}
$$

(ii) there exist a nontrivial $\mathbb{R}$-linear functional $L: X \rightarrow \mathbb{R}$, a homomorphism $\psi$ of the multiplicative semigroup of nonnegative real numbers into $(S, \circ)$, $z \in Z(\psi([0, \infty))), s, t \in S, k \in K \backslash\{0\}$ and $l \in \mathbb{R}$ such that

$$
\begin{aligned}
& F(x)= \begin{cases}s \circ t \circ \psi(L(x)+l) & \text { whenever } \\
s \circ t \circ z & \text { otherwise, }\end{cases} \\
& G(x)=k \max \{L(x)+l, 0\} \quad \text { for } \quad x \in X,
\end{aligned}
$$




$$
\begin{aligned}
& H(x)= \begin{cases}t \circ \psi(L(x)+l) & \text { whenever } \\
t \circ z & \text { otherwise, }\end{cases} \\
& K(x)= \begin{cases}s \circ \psi(L(k x)+l) & \text { whenever } \\
s \circ z & \text { otherwise. }\end{cases}
\end{aligned}
$$

Proof. Assume that a quadruple $(F, G, H, K)$ satisfies equation (6). Then, according to Proposition 6 there exist $x_{0} \in X, s, t \in S, k \in K \backslash\{0\}$ and functions $f: X \rightarrow S, g: X \rightarrow K$ such that the pair $(f, g)$ satisfies (5) and (38)-(41) hold. Moreover, as $G$ satisfies one of the conditions $\left(C_{1}\right)-\left(C_{3}\right)$, so does $g$; and as $F$ and $G$ are nonconstant, so are $f$ and $g$. Hence one of the conditions $(a)$ or $(b)$ of Proposition 3 holds. Therefore, taking $l:=1-L\left(x_{0}\right)$, we obtain $(i)$ or $($ ii $)$, respectively.

Since the converse is easy to check, the proof is completed.

Open Access. This article is distributed under the terms of the Creative Commons Attribution License which permits any use, distribution, and reproduction in any medium, provided the original author(s) and the source are credited.

\section{References}

[1] Aczél, J., Dhombres, J.: Functional Equations in Several Variables. Encyclopedia of Mathematics and its Applications. 31. Cambridge University Press, Cambridge (1989)

[2] Baron, K.: On the continuous solutions of the Gołąb-Schinzel equation. Aequationes Math. 38, 155-162 (1989)

[3] Brillouët, N., Dhombres, J.: Équations fonctionnelles et recherche de sousgroupes. Aequationes Math. 31, 253-293 (1986)

[4] Brzdȩk, J.: Subgroups of the group $Z_{n}$ and a generalization of the Gołąb-Schinzel functional equation. Aequationes Math. 43, 59-71 (1992)

[5] Brzdȩk, J.: A generalization of addition formulae. Acta Math. Hungar. 101, 281291 (2003)

[6] Brzdȩk, J.: Gołąb-Schinzel equation and its generalizations. Aequationes Math. 70, 14-24 (2005)

[7] Brzdȩk, J.: A remark on solutions of a generalization of the addition formulae. Aequationes Math. 71, 288-293 (2006)

[8] Brzdęk, J.: On the quotient stability of a class of functional eqautions. Nonlinear Anal. 71, 4396-4404 (2009)

[9] Brzdȩk, J.: On stability of a family of functional equations. Acta Math. Hungar. 128, 139-149 (2010)

[10] Charifi, A., Bouikhalene, B., Kabbaj, S., Rassias, M.M.: On the stability of the pexiderized Gołąb-Schinzel equation. Comput. Math. Appl. 59, 3193-3202 (2010)

[11] Chudziak, J.: On a functional inequality related to the stability problem for the Gob-Schinzel equation. Publ. Math. Debrecen 67, 199-208 (2005)

[12] Chudziak, J.: Approximate solutions of the Gołąb-Schinzel equation. J. Approx. Theory 136, 21-25 (2005)

[13] Chudziak, J.: Stability of the generalized Gołąb-Schinzel equation. Acta Math. Hungar. 113, 133-144 (2006) 
[14] Chudziak, J.: Semigroup-valued solutions of the Gołąb-Schinzel type functional equation. Abh. Math. Sem. Univ. Hamburg 76, 91-98 (2006)

[15] Chudziak, J.: Approximate solutions of the generalized Gob-Schinzel equation. J. Inequal. Appl., Art. ID 89402 1-8 (2006)

[16] Chudziak, J.: Associative solutions of a composite generalization of addition formulae. Acta Math. Hungar. 117, 141-152 (2007)

[17] Chudziak, J.: Stability problem for the Gołąb-Schinzel type functional equations. J. Math. Anal. Appl. 339, 454-460 (2008)

[18] Chudziak, J., Tabor, J.: On the stability of the Gołąb-Schinzel functional equation. J. Math. Anal. Appl. 302, 196-200 (2005)

[19] Jabłońska, E.: Functions having the Darboux property and satisfying some functional equation. Colloq. Math. 114, 113-118 (2009)

[20] Jabłońska, E.: Solutions of a Gołąb-Schinzel-type functional equation bounded on 'big' sets in an abstract sense. Bull. Aust. Math. Soc. 81, 430-441 (2010)

[21] Jabłońska, E.: Christensen measurable solutions of some functional equation. Nonlinear Anal. 72, 2465-2473 (2010)

[22] Jabłońska, E.: Continuous on rays solutions of an equation of the Gołąb-Schinzel type. J. Math. Anal. Appl. 375, 223-229 (2011)

[23] Jabłońska, E.: The Pexiderized Gołąb-Schinzel functional equation. J. Math. Anal. Appl. 381, 565-572 (2011)

[24] Kominek, Z., Kuczma, M.: Theorems of Bernstein-Doetsch, Piccard and Mehdi and semilinear topology. Arch. Math. (Basel) 52, 595-602 (1989)

[25] Mureńko, A.: On solutions of a common generalization of the Gołąb-Schinzel functional equation and of the addition formulae. J. Math. Anal. Appl. 341, 1236$1240(2008)$

[26] Mureńko, A.: On the general solution of a generalization of the Gołąb-Schinzel equation. Aequationes Math. 77, 107-118 (2009)

Jacek Chudziak

Department of Mathematics

University of Rzeszów

Rejtana $16 \mathrm{C}$

35-959 Rzeszów

Poland

e-mail: chudziak@ur.edu.pl

Received: May 17, 2013

Revised: July 29, 2013 